\title{
Effects of aromatic amino acids, phenylacetate and phenylpropionate on fermentation of xylan by species of rumen anaerobic bacteria
}

\author{
A. Y. Guliye ${ }^{1 *}$ and R. J. Wallace ${ }^{2}$ \\ ${ }^{1}$ Department of Animal Science, Egerton University, P. O. Box 536-20115, Egerton, Kenya. \\ ${ }^{2}$ Rowett Institute of Nutrition and Health, Bucksburn, Aberdeen AB21 9SB, UK.
}

Accepted 2 September, 2013

\begin{abstract}
This study determined the effects of aromatic amino acids (AA) and phenyl acids (phenylacetic acid and phenylpropionic acid) on fermentation of xylan by selected rumen bacteria. The ruminal bacteria were grown in a defined medium containing oat spelts xylan as the sole energy source, plus one of the following N sources: ammonia (no AA); ammonia plus a complete mixture of 20 AA commonly found in protein (CAA); ammonia plus complete AA mixture minus aromatic AA (MAR); ammonia plus phenyl acids (no AA+PA); ammonia plus complete AA mixture without aromatic AA plus phenyl acids (MAR+PA). There was varied growth response, with Fibrobacter succinogenes S85, Ruminococcus flavefaciens FD1, and Butyrivibrio fibrisolvens JW11 not being able to grow. The supply of CAA mixture significantly $(P<0.05)$ increased acetate production in all species, in comparison with no AA treatment, by 54, 30, 97 and $33 \%$ in Prevotella bryantii $B_{1} 4$, Ruminococcus albus SY3, R. flavefaciens 17 and Pseudobutyrivibrio xylanivorans JK170 cultures, respectively. The addition of phenyl acids significantly $(P<0.05)$ increased acetate yields by 34,8 and $5 \%$ in $P$. bryantii $B_{1} 4, R$. albus SY3 and $P$. xylanivorans JK170 cultures, respectively. Sugar utilization by all the species significantly $(P<0.05)$ increased by 42 and $77 \%$ when CAA mixture was provided. The deletion of aromatic AA from the complete AA mixture caused a significant $(P<0.05)$ reduction in sugar utilization in $P$. bryantii $\mathrm{B}_{1} 4$ and $P$. xylanivorans $\mathrm{JK} 170$ by 25 and $10 \%$, respectively. Phenyl acids addition increased sugar utilization by 4.8 and $5.1 \%$ in $R$. albus SY3 and $R$. flavefaciens 17 , respectively. The supply of CAA mixture significantly $(P<0.05)$ increased microbial yield in $P$. bryantii $\mathrm{B}_{1} 4, R$. albus $S Y 3, R$. flavefaciens 17 and $P$. xylanivorans JK170 species. It is concluded that supplementation of complete mixture of AA is beneficial in stimulating bacterial growth in the rumen.
\end{abstract}

Key words: Aromatic amino acids, phenylacetic acid, phenylpropionic acid, rumen bacteria, xylan.

\section{INTRODUCTION}

Complete mixtures of all amino acids $(\mathrm{AA})$ stimulate the rate of fermentation and the microbial growth yield (Cotta and Russell, 1982; Chen et al., 1987; Cruz Soto et al., 1993). However, aromatic AA have been known to limit the growth of microbes and xylan fermentation by mixed rumen micro-organisms (Kajikawa et al., 2002; Atasoglu et al., 2003; Guliye et al., 2005). Phenylalanine, one of the aromatic $\mathrm{AA}$, has been suggested to be essential for the growth of cellulolytic bacterial species, including Ruminococcus (Allison 1965) and Fibrobacter succinogenes (Allison, 1965; Atasoglu et al., 2001). Fungal strains of Neocallimastix spp., provided with cellobiose as energy source, also showed reduced growth when aromatic AA were omitted from a complete 
mixture of 18 AA (Onoda et al., 1996), and achieved maximum growth when a complete mixture were supplied (Orpin and Greenwood, 1986; Onoda et al., 1996; Guliye and Wallace, 2007).

The precursors for the synthesis of aromatic AA, phenylacetic acid (PAA) and phenylpropionic acid (PPA), can be beneficial to mixed populations of rumen bacteria and protozoa (Scott et al., 1964; Kristensen, 1974; Amin and Onodera, 1997). The phenyl acids (PAA and PPA) have also been reported to stimulate growth and to improve cellulose degradation by Ruminococcus albus (Allison, 1965; Hungate and Stack, 1982; Stack et al., 1983; Stack and Cotta, 1986; Morrison et al., 1990).

Fibrous plant roughages, a significant portion being hemicellulose, form the bulk of ruminant livestock diets. Xylan is a predominant polymer in hemicellulose. Much of xylan degradation occurs in the rumen and is carried out by a number of ruminal organisms, including bacteria. The key rumen bacteria involved in xylan degradation include Butyrivibrio fibrisolvens and Prevotella ruminicola (Dehority, 2003), Prevotella bryantii (Miyazaki et al., 1997), Pseudobutyrivibrio xylanivorans (Kopecny et al., 2003), and the cellulolytic species Fibrobacter succinogenes, $R$. albus, and $R$. flavefaciens (Hespell and Whitehead, 1990). Therefore, understanding the AA and phenyl acids effects on rumen bacteria may be important to ruminal xylan fermentation efficiency. However, there are limited pure culture data published on the possible effect(s) of aromatic AA, as a group, on xylan utilization and growth of the main xylanolytic organisms in the rumen. The aim of the present study was to determine the effects of aromatic AA or their derivative phenyl acids (PA) on the fermentation of xylan by selected rumen anaerobic bacteria.

\section{MATERIALS AND METHODS}

\section{Organisms}

The rumen bacteria used in this study were obtained from the culture collection maintained at the Rowett Research Institute (currently Rowett Institute of Nutrition and Health), Aberdeen (UK), and were selected based on their presumed ability to degrade and utilize xylan (oat spelts xylan), the substrate used as energy source. Therefore, the following organisms were used: $F$. succinogenes $\mathrm{S} 85, R$. flavefaciens $17, R$. flavefaciens FD1, $R$. albus SY3, $B$. fibrisolvens JW11, Pseudobutyrivibrio. xylanivorans JK170 and $P$. bryantii $\mathrm{B}_{1} 4$.

\section{Media and growth conditions}

The organisms, obtained from stock cultures, were initially grown overnight under anaerobic conditions at $39^{\circ} \mathrm{C}$ in $10 \mathrm{ml}$ of $\mathrm{M} 2$ medium (Hobson, 1969). The organisms were then inoculated $(5 \%$, $\mathrm{vol} / \mathrm{vol}$ ) into $100 \mathrm{ml}$ Wheaton bottles containing the defined medium of Hungate and Stack (1982), modified to include $0.3 \%(w / v)$ oat spelts xylan (OSX) (Sigma, Steinheim-Germany) as the sole energy source, to enable them adapt to the xylan. The cultures were then grown overnight at $39^{\circ} \mathrm{C}$ and checked for species conformation through gram staining. These fresh cultures were then used to inoculate $(5 \%$, vol/vol) triplicate Wheaton bottles containing $100 \mathrm{ml}$ of fresh modified Hungate and Stack medium, with various additions according to treatment specifications. The wheaton bottles were then placed in a shaking water bath at $39^{\circ} \mathrm{C}$ and the cultures were incubated for up to $36 \mathrm{~h}$.

\section{Treatments}

There were five treatments: ammonia only (no AA); ammonia plus complete mixture of 20 AA commonly found in protein (CAA); ammonia plus the complete AA mixture but with aromatic AA omitted (MAR); ammonia plus phenyl acids (no AA+PA); ammonia plus the complete AA mixture with aromatic AA omitted, plus phenyl acids (MAR+PA). The concentration of ammonia, supplied in form of $\mathrm{NH}_{4} \mathrm{Cl}$ to all treatments, was $10 \mathrm{mmol} \mathrm{L}^{-1}$. The AAs were added to a final concentration of $0.25 \mathrm{~g} \mathrm{~L}^{-1}$ for each AA. The PAA and PPA were added, where applicable, to a final concentration of 10 and 25 $\mathrm{mmol} \mathrm{L}^{-1}$, respectively (Stack and Cotta, 1986; Stack et al., 1983). Treatments where PAA and PPA were not supplemented had the same volume of distilled water added instead. The $\mathrm{N}$ sources and phenyl acids were added to the media during preparation.

\section{Experimental measurements}

The Wheaton bottles were periodically shaken and samples obtained anaerobically via the septum using sterile needles and syringes at the start $(10 \mathrm{ml})$, during $(2 \mathrm{ml}$, at various time points) and at the end of the experiment $(10 \mathrm{ml})$, when stationary phase had been reached. Two millilitres of the collected samples were solubilized in $0.5 \mathrm{~mol} \mathrm{I}^{-1} \mathrm{NaOH}$ and used to determine protein using the Bradford assay (Bradford, 1976). The remainder of the $0 \mathrm{~h}$ and stationary phase samples (18 or $24 \mathrm{~h}$ ) were kept frozen and used later for the determination of volatile fatty acids (VFA), ammonia, sugar utilization and total cell $\mathrm{N}(\mathrm{TCN})$ as described by Guliye et al. (2005).

\section{Calculations and statistical analysis}

The results are all means derived from the analysis of triplicate cultures. Net values were calculated as 18 or $24 \mathrm{~h}$ concentration - 0 h concentration for VFA, ammonia, sugar utilization and TCN. Microbial yield (expressed as $\mathrm{g} \mathrm{N} \mathrm{kg}^{-1}$ sugar utilised) was calculated from the net microbial $\mathrm{N}$ synthesis (TCN) divided by the net sugar utilization. The data were analysed by ANOVA, with $\mathrm{N}$ source (no $A A, C A A, M A R$ ) and phenyl acids addition (no AA+PA and MAR+PA) as treatment effects, using Genstat program release 6.1 (Lawes Agricultural Trust, Rothamsted Herts, UK). Multiple mean comparisons were done using Fisher's comparison.

\section{RESULTS}

\section{Bacterial growth}

All species from the stock cultures grew well in the M2 medium. However, when transferred into the initial modified Hungate and Stack medium, which was used as inoculum, not all cultures grew actively. While $P$. bryantii $\mathrm{B}_{1} 4, R$. albus SY3, $R$. flavefaciens 17 and $P$. xylanivorans JK170 strains grew well, $F$. succinogenes S85, $R$. flavefaciens FD1 and B. fibrisolvens JW11 were less 
Prevotella bryantii $\mathrm{B}_{1} 4$

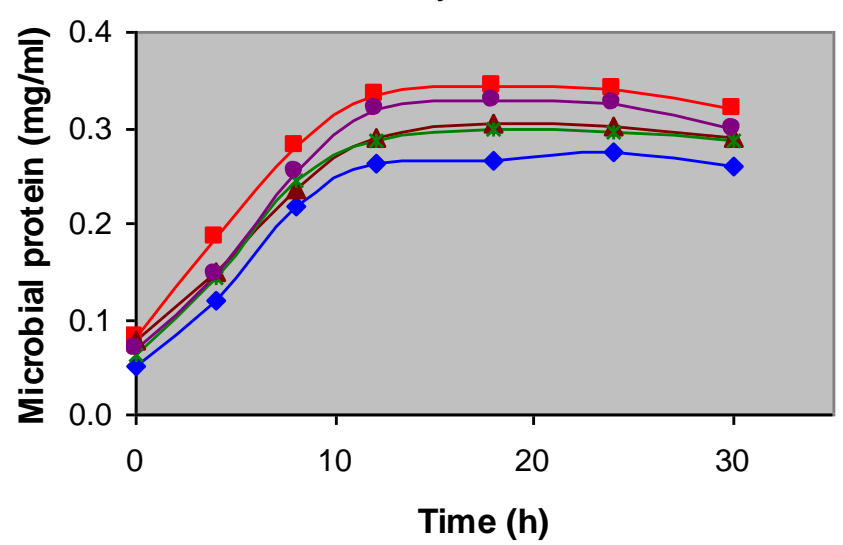

Ruminococcus flavefaciens 17

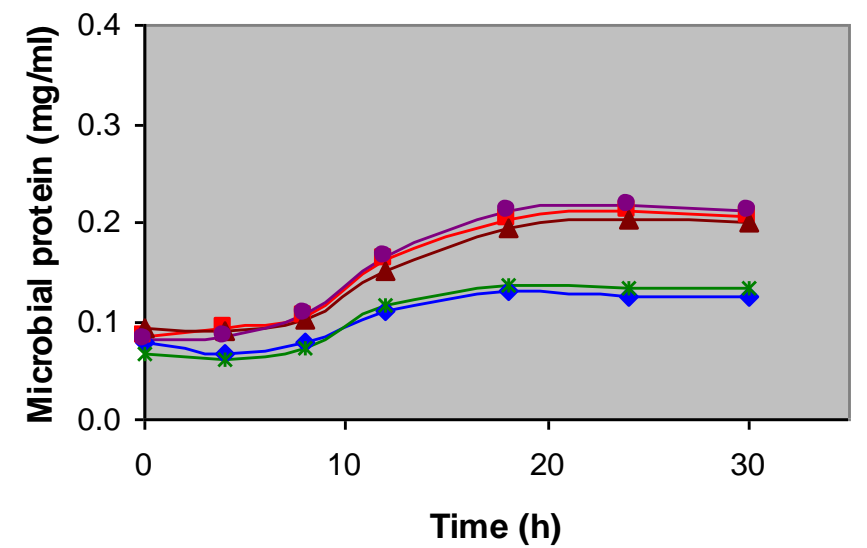

Ruminococcus albus SY3

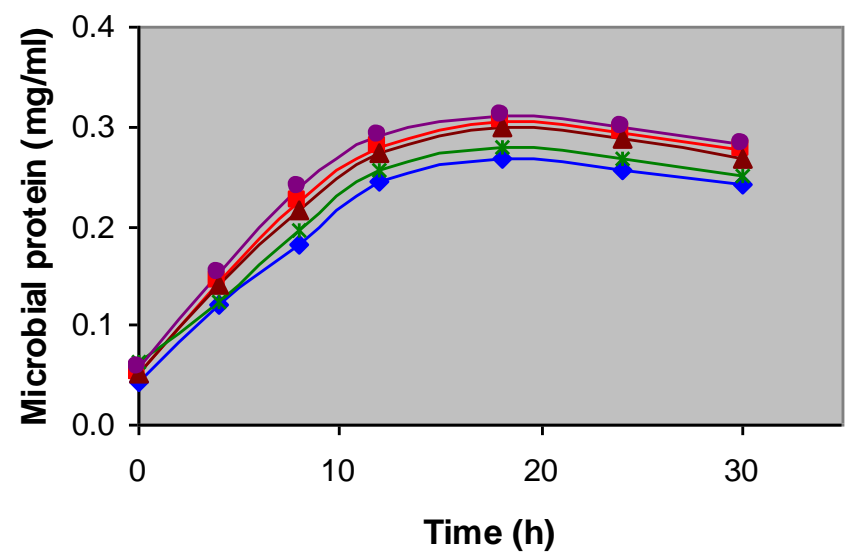

Pseudobutyrivibrio xylanivorans JK170

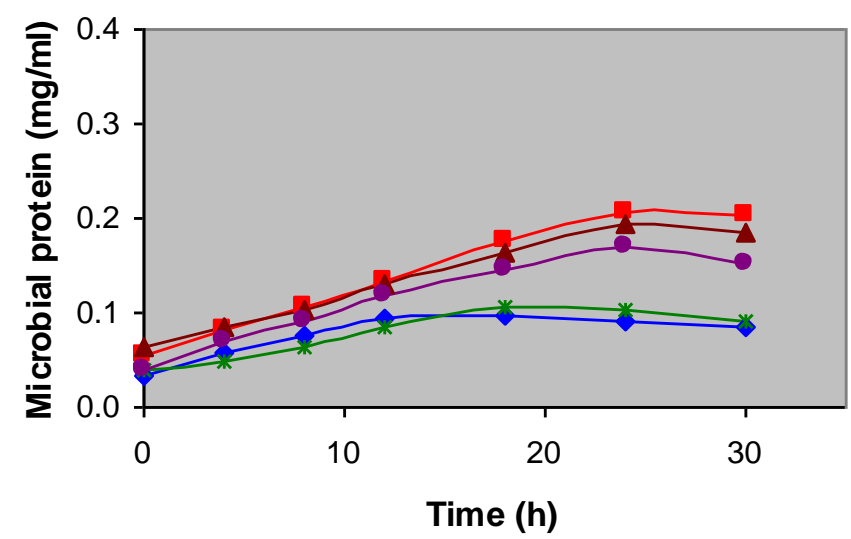

Figure 1. Growth of ruminal bacteria on oat spelts xylan in the presence of: ammonia only with no amino acids - AA ( $\bullet$ ), complete AA mixture - CAA (ם), complete AA mixture minus aromatic AA - MAR ( $\mathbf{A})$, ammonia only plus phenyl acids - no AA+PA (*), and complete AA mixture minus aromatic AA plus phenyl acids - MAR+PA (@). Data points represent mean values from triplicate cultures.

active in their growth. When transferred into the final growth medium of modified Hungate and Stack, $P$. bryantii $\mathrm{B}_{1} 4, R$. albus SY3, $R$. flavefaciens 17 and $P$. xylanivorans JK170 strains all grew, the first two strains having reached stationary phase at $18 \mathrm{~h}$ while the latter two reached at $24 \mathrm{~h}$ (Figure 1). $P$. bryantii $\mathrm{B}_{1} 4$ showed the highest growth, closely followed by $R$. albus SY3. However, F. succinogenes S85, R. flavefaciens FD1, and $B$. fibrisolvens JW11 failed to grow in the modified Hungate and Stack final growth medium (Figure 2). The growth observed earlier with the modified Hungate and Stack inoculum media may have been due to nutrients, especially energy source, transferred in the M2 medium.

\section{Volatile fatty acids (VFA) and ammonia}

Acetate was the major VFA produced by all the species that were able to grow (Table 1). In addition, $P$. xylanivorans JK170 also produced significant amounts of butyrate. None of the species produced propionate or caproate irrespective of treatments.

The supply of complete AA mixture (CAA treatment) significantly $(P<0.05)$ increased acetate production in all the species, in comparison with no AA treatment, by 54 , 30, 97 and $33 \%$ in $P$. bryantii $\mathrm{B}_{1} 4, R$. albus SY3, $R$. flavefaciens 17 and $P$. xylanivorans JK170 cultures, respectively. While valerate was not significantly influenced by the addition of complete mixtures of $A A$ in $R$. albus SY3 and $R$. flavefaciens 17 , there was 13 fold increase in iso-butyrate by $P$. bryantii $\mathrm{B}_{1} 4$ (Table 1 ). Also, butyrate production by $P$. xylanivorans JK170 increased significantly $(P<0.05)$ by fivefold in complete AA mixtures (Table 1).

The deletion of aromatic AA from a complete mixture (MAR treatment) did not influence acetate yields in $R$. flavefaciens 17 and $P$. xylanivorans JK170, however, there was 24 and $5 \%$ decline $(P<0.05)$ observed in $P$. bryantii $\mathrm{B}_{1} 4$ 
Fibrobacter succinogenes S85

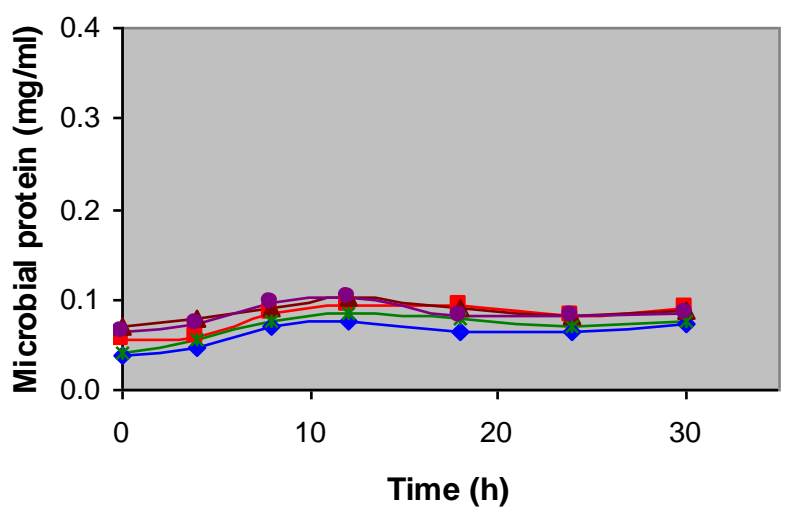

Ruminococcus flavefaciens FD1

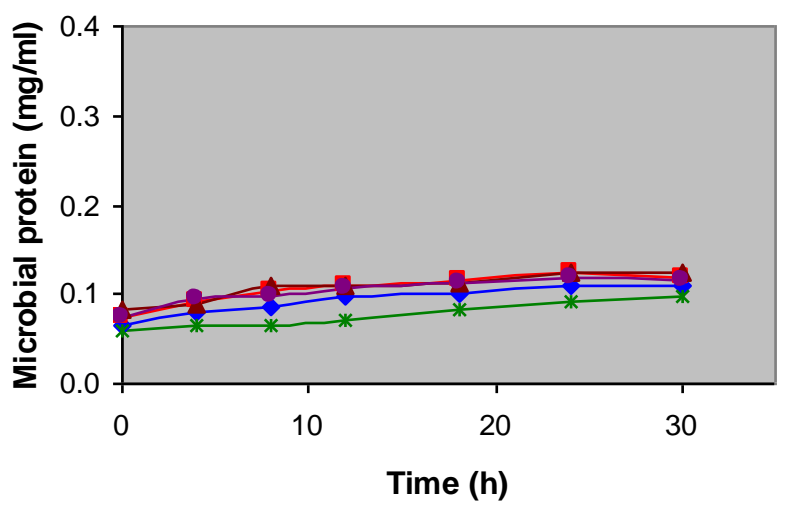

Butyrivibrio fibrisolvens JW11

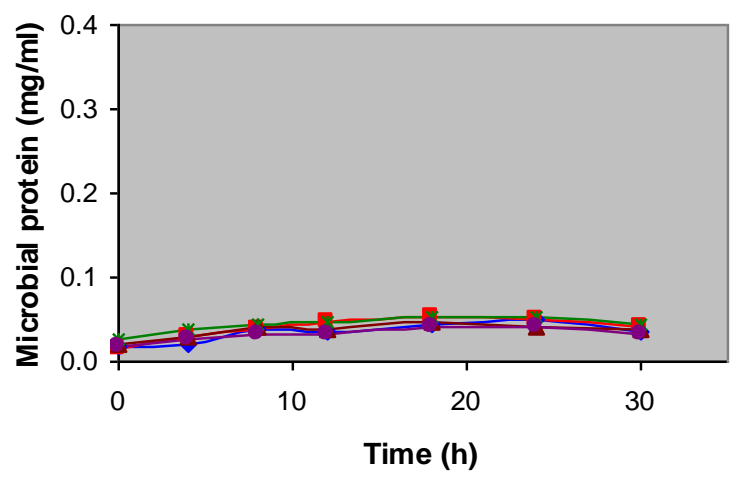

Figure 2. Growth of ruminal bacteria on oat spelts xylan in the presence of: ammonia only with no amino acids - AA ( $\bullet$ ), complete AA mixture - CAA $(\square)$, complete AA mixture minus aromatic AA - MAR $(\mathbf{\Delta})$, ammonia only plus phenyl acids - no $\mathrm{AA}+\mathrm{PA}(*)$, and complete AA mixture minus aromatic AA plus phenyl acids - MAR+PA (๑). Data points represent mean values from triplicate cultures.

Table 1. Effects of aromatic AA and phenyl acids (PA) on volatile fatty acids (VFA) production by ruminal bacteria fermenting xylan.

\begin{tabular}{lllllccc}
\hline \multirow{2}{*}{ Bacterial species } & \multicolumn{7}{c}{ Treatments } \\
\cline { 2 - 7 } & VFA $\left(\mathbf{m m o l ~ I} \mathbf{I}^{-1}\right)$ & no AA & CAA & MAR & no AA +PA & MAR +PA & SEM \\
\hline Prevotella & Acetate & $3.11^{\mathrm{a}}$ & $4.78^{\mathrm{b}}$ & $3.64^{\mathrm{c}}$ & $3.32^{\mathrm{d}}$ & $4.72^{\mathrm{b}}$ & 0.06 \\
Bryantii $\mathrm{B}_{14}$ & iso-butyrate & $0.01^{\mathrm{a}}$ & $0.13^{\mathrm{b}}$ & $0.11^{\mathrm{b}}$ & $0.00^{\mathrm{a}}$ & $0.06^{\mathrm{c}}$ & 0.01 \\
Ruminococcus & Acetate & $4.83^{\mathrm{a}}$ & $6.25^{\mathrm{b}}$ & $5.92^{\mathrm{c}}$ & $5.01^{\mathrm{a}}$ & $6.39^{\mathrm{b}}$ & 0.04 \\
Albus SY3 & Valerate & 0.00 & 0.02 & 0.01 & 0.01 & 0.01 & 0.01 \\
Ruminococcus & Acetate & $2.35^{\mathrm{a}}$ & $4.64^{\mathrm{b}}$ & $4.58^{\mathrm{b}}$ & $2.72^{\mathrm{a}}$ & $4.59^{\mathrm{b}}$ & 0.25 \\
Flavefaciens 17 & Valerate & $0.01^{\mathrm{a}}$ & $0.04^{\mathrm{a}}$ & $0.01^{\mathrm{a}}$ & $0.04^{\mathrm{a}}$ & $0.12^{\mathrm{b}}$ & 0.01 \\
Pseudobutyrivibrio & Acetate & $3.20^{\mathrm{a}}$ & $4.25^{\mathrm{b}, \mathrm{d}}$ & $4.17^{\mathrm{b}}$ & $3.68^{\mathrm{c}}$ & $4.37^{\mathrm{d}}$ & 0.05 \\
Xylanivorans JK170 & Butyrate & $0.83^{\mathrm{a}}$ & $3.88^{\mathrm{b}}$ & $3.41^{\mathrm{c}}$ & $0.96^{\mathrm{a}}$ & $3.25^{\mathrm{c}}$ & 0.07 \\
\hline
\end{tabular}

Treatments were: no $A A=$ ammonia only, $C A A=$ ammonia + complete mixture of 20 amino acids, $M A R=$ ammonia + complete mixture minus aromatic amino acids, no $A A+P A=$ ammonia only plus phenyl acids, and MAR+PA = ammonia + complete mixture minus aromatic amino acids plus phenyl acids. Phenyl acids (PA) were phenylacetic acid $\left(10 \mathrm{mmol} \mathrm{L}{ }^{-1}\right)$ and phenylpropionic acid $\left(25 \mathrm{mmol} \mathrm{L}^{-1}\right)$. Amino acids were added at $0.25 \mathrm{~g} \mathrm{~L}^{-1}$ for each amino acid. Xylan (sugar) was added at $3 \mathrm{~g} \mathrm{~L}^{-1}$. Values are means calculated from triplicate cultures. Means in the same row with different superscripts differ $(P<0.05)$. Propionic, butyric (except for $P$. xylanivorans JK170) and caproic acids were not detected in all treatments. Valeric, iso-butyric (except for P. bryantii B14) and iso-valeric acids appeared as traces.

and $R$. albus SY3, respectively. The addition of phenyl acids, PAA and PPA, to ammonia (no AA treatment) significantly $(P<0.05)$ increased acetate production by 7 and $15 \%$ increase in $P$. bryantii $B_{1} 4$ and $P$. xylanivorans 
Table 2. Effects of aromatic $A A$ and phenyl acids (PA) on ammonia ( $\mathrm{mg} \mathrm{l}^{-1}$ ) production by ruminal bacteria fermenting xylan.

\begin{tabular}{lcccccc}
\hline \multirow{2}{*}{ Organism } & \multicolumn{5}{c}{ Treatment } \\
\cline { 2 - 7 } & no AA & CAA & MAR & no AA+PA & MAR+PA & SEM \\
\hline Prevotella bryantii $\mathrm{B}_{1} 4$ & $-24.3^{\mathrm{a}}$ & $85.5^{\mathrm{b}}$ & $73.6^{\mathrm{c}}$ & $-31.1^{\mathrm{a}}$ & $60.2^{\mathrm{d}}$ & 2.22 \\
Ruminococcus albus SY3 & $-43.9^{\mathrm{a}}$ & $54.7^{\mathrm{b}}$ & $46.2^{\mathrm{c}}$ & $-48.0^{\mathrm{a}}$ & $36.3^{\mathrm{d}}$ & 1.19 \\
Ruminococcus flavefaciens 17 & $-24.4^{\mathrm{a}}$ & $35.4^{\mathrm{b}}$ & $29.8^{\mathrm{c}}$ & $-26.5^{\mathrm{a}}$ & $33.9^{\mathrm{b}}$ & 0.56 \\
Pseudobutyrivibrio xylanivorans JK170 & $-19.9^{\mathrm{a}}$ & $32.9^{\mathrm{b}}$ & $31.1^{\mathrm{b}, \mathrm{c}}$ & $-21.4^{\mathrm{a}}$ & $29.1^{\mathrm{c}}$ & 0.54 \\
\hline
\end{tabular}

Treatments were: no $A A=$ ammonia only, $C A A=$ ammonia + complete mixture of 20 amino acids, MAR = ammonia + complete mixture minus aromatic amino acids, no $\mathrm{AA}+\mathrm{PA}=$ ammonia only plus phenyl acids, and MAR+PA = ammonia + complete mixture minus aromatic amino acids plus phenyl acids. Phenyl acids (PA) were phenylacetic acid $\left(10 \mathrm{mmol} \mathrm{I}^{-1}\right)$ and phenylpropionic acid $\left(25 \mathrm{mmol} \mathrm{L}^{-1}\right)$. Amino acids were added at $0.25 \mathrm{~g} \mathrm{~L}^{-1}$ for each amino acid. Xylan (sugar) was added at $3 \mathrm{~g} \mathrm{~L}^{-1}$. Values are means calculated from triplicate cultures. Means in the same row with different superscripts differ $(P<0.05)$.

JK170, respectively (Table 1). Also, phenyl acids addition to a complete mixture of $A A$ with aromatic $A A$ omitted (MAR) (MAR) significantly $(P<0.05)$ increased acetate yields by 34,8 and $5 \%$ in $P$. bryantii $\mathrm{B}_{1} 4, R$. albus SY3 and $P$. xylanivorans JK170 cultures, respectively. In all species, acetate production was not significantly different between $\mathrm{CAA}$ and MAR+PA treatments.

Although butyrate production decreased $(P<0.05)$ by about $12 \%$ as a result of the deletion of aromatic AA from a complete AA mixture in $P$. xylanivorans JK170, the addition of phenyl acids to a medium that contained complete AA mixtures with aromatic AA omitted (MAR+PA treatment) did not significantly influence butyrate production (Table 1). Butyrate yields by $P$. xylanivorans JK170 was significantly lower in MAR+PA treatment, compared to CAA, despite the addition of phenyl acids. Valerate yields increased $(P<0.05)$ over ten fold in $R$. flavefaciens 17 due to addition of phenyl acids, in comparison with MAR treatment (Table 1), however, it was not influenced by the deletion of aromatic AA from a complete AA mixture in $R$. albus SY3 and $R$. flavefaciens 17. The addition of $A A$, whether complete or with aromatic AA omitted, significantly $(P<0.05)$ increased isobutyrate yields by $P$. Bryantii $\mathrm{B}_{1} 4$, in comparison with ammonia only (no AA) treatment (Table 1). However, compared to MAR treatment, there was almost $45 \%$ decline in iso-butyrate production by $P$. bryantii $\mathrm{B}_{1} 4$ when phenyl acids were provided (MAR+PA treatment).

The addition of $A A$, whether the complete mixture or minus aromatic $A A$, resulted in increased ammonia yields by all the organisms, the increase being highest in the treatment where complete mixtures of $A A(C A A)$ were supplemented (Table 2). The yields of ammonia produced declined in all the species when aromatic AAs were omitted from a complete mixture of AA. The decrease, significant at $P<0.05$, was 14,16 and $16 \%$ for $P$. bryantii $\mathrm{B}_{1} 4, R$. albus $\mathrm{SY} 3$ and $R$. flavefaciens 17 , respectively.

Phenyl acids added to a medium that contained only ammonia as sole $\mathrm{N}$ source had no significant influence on ammonia production. Conversely, the addition of phenyl acids to complete AA mixtures with aromatic AA omitted (MAR+PA treatment) led to a signi-ficant $(P<0.05)$ decrease in ammonia in most species, in comparison with MAR treatment. The decrease was 18 , 21 and $6 \%$ for $P$. bryantii $\mathrm{B}_{1} 4, R$. albus SY3, and $P$. xylanivorans JK170, respectively. However, there was a $14 \%$ increase in ammonia production by $R$. flavefaciens 17. The net ammonia in the treatments where $A A$ were not provided (no $A A$ and no $A A+P A$ ) had negative values (Table 2), since the microorganisms utilised the ammonia in the medium, which was the only source of $\mathrm{N}$ provided.

\section{Sugar utilization}

The utilization of xylan (OSX), as the sole energy source, by the bacterial species is presented in Table 3 . The amount of sugar (xylan) utilised was greatest for $P$. bryantii $\mathrm{B}_{1} 4$ and $R$. albus $\mathrm{SY} 3$. The addition of $\mathrm{AA}$, whether the complete mixture or minus aromatic $A A$, stimulated sugar utilization. Compared with no AA treatment, sugar utilization by all the species significantly $(P<0.05)$ increased by between 42 and $77 \%$ when a complete mixture of AA was provided. The deletion of aromatic AA from the complete AA mixture caused a significant $(P<0.05)$ reduction in sugar utilization only in $P$. bryantii $\mathrm{B}_{1} 4$ and $P$. xylanivorans JK170 by 25 and $10 \%$, respectively).

The addition of phenyl acids to a medium that contained ammonia as the only source of $\mathrm{N}$ (no AA vs no $A A+P A$ treatments) did not significantly increase sugar (xylan) utilization, except in $P$. bryantii $\mathrm{B}_{1} 4$ where a $25 \%$ increase $(P<0.05)$ was observed (Table 3$)$. However, compared to the MAR treatment (that is, complete AA mixture with aromatic AA omitted), addition of phenyl acids significantly $(P<0.05)$ increased sugar utilization only in $P$. bryantii $\mathrm{B}_{1} 4$ and $R$. albus SY 3 by 20 and $13 \%$, respectively. Compared to CAA treatment, the addition of phenyl acids to a medium that had complete AA mixture with aromatic AA omitted (MAR treatment) showed increased utilization of sugar by $4.8 \%$ and $5.1 \%$ in $R$. albus SY3 and $R$. flavefaciens 17, respectively, which was however, significant $(P<0.05)$ only for the former. 
Table 3. Effects of aromatic AA and phenyl acids (PA) on xylan utilization by species of rumen bacteria.

\begin{tabular}{lccccccc}
\hline \multirow{2}{*}{ Organism } & \multicolumn{7}{c}{ Treatments } \\
\cline { 2 - 8 } & Sugar utilised & no AA & CAA & MAR & no AA +PA & MAR +PA $^{1}$ & SEM \\
\hline Prevotella & $\mathrm{g} / \mathrm{L}$ & $1.21^{\mathrm{a}}$ & $1.95^{\mathrm{b}}$ & $1.50^{\mathrm{c}}$ & $1.51^{\mathrm{c}}$ & $1.79^{\mathrm{d}}$ & 0.03 \\
Bryantii $\mathrm{B}_{1} 4$ & $\%$ & 40 & 65 & 50 & 50 & 60 & \\
Ruminococcus & $\mathrm{g} / \mathrm{L}$ & $1.25^{\mathrm{a}}$ & $1.68^{\mathrm{b}}$ & $1.60^{\mathrm{b}}$ & $1.32^{\mathrm{a}}$ & $1.76^{\mathrm{c}}$ & 0.03 \\
Albus SY3 & $\%$ & 42 & 56 & 53 & 44 & 59 & \\
Ruminococcus & $\mathrm{g} / \mathrm{L}$ & $0.79^{\mathrm{a}}$ & $1.17^{\mathrm{b}, \mathrm{c}}$ & $1.11^{\mathrm{b}}$ & $0.81^{\mathrm{a}}$ & $1.23^{\mathrm{b}}$ & 0.02 \\
Flavefaciens 17 & $\%$ & 26 & 39 & 37 & 27 & 41 & \\
Pseudobutyrivibrio & $\mathrm{g} / \mathrm{L}$ & $0.62^{\mathrm{a}}$ & $1.07^{\mathrm{b}}$ & $1.00^{\mathrm{c}}$ & $0.65^{\mathrm{a}}$ & $0.95^{\mathrm{c}}$ & 0.02 \\
Xylanivorans JK170 & $\%$ & 21 & 36 & 33 & 22 & 32 & \\
\hline
\end{tabular}

Treatments were: no $A A=$ ammonia only, $C A A=$ ammonia + complete mixture of 20 amino acids, $M A R=$ ammonia + complete mixture minus aromatic amino acids. Phenyl acids (PA) were phenylacetic acid $\left(10 \mathrm{mmol} \mathrm{L}^{-1}\right)$ and phenylpropionic acid $\left(25 \mathrm{mmol}^{-1}\right)$. Amino acids were added at $0.25 \mathrm{~g} \mathrm{~L}^{-1}$ for each amino acid. Xylan (sugar) was added at $3 \mathrm{~g} \mathrm{~L}^{-1}$. Values are means calculated from triplicate cultures. Means in the same row with different superscripts differ $(P<0.05)$.

\section{Microbial yield}

Microbial yield (g TCN kg-1 sugar utilised), calculated from the initial and final concentrations of cell protein and sugar (xylan), is presented in Figure 3. The yields recorded in the present study ranged between 27 and 31 for $P$. bryantii $\mathrm{B}_{1} 4$; between 27 to about 30 for $R$. albus SY3; between 21 and 28 for $R$. flavefaciens 17; and between 17 and 27 for $B$. fibrisolvens 170 (Figure 3).

The addition of a complete $A A$ mixture to a medium that had only ammonia as $N$ source (no AA vs CAA treatments) significantly $(P<0.05)$ increased microbial yield from 27.4 to $32.0,27.2$ to $29.3,20.8$ to 27.9 , and 16.9 to $27.4 \mathrm{~g} \mathrm{~kg}^{-1}$ for $P$. bryantii $\mathrm{B}_{1} 4, R$. albus SY3, $R$. flavefaciens 17 and $P$. xylanivorans JK170 species, respectively. The deletion of aromatic AA from a complete AA mixture (MAR treatment) did not significantly decrease microbial yields in all species, except for $P$. bryantii $\mathrm{B}_{1} 4$ which showed about $7 \%$ decrease in yields.

The addition of phenyl acids to a medium that contained only ammonia as $\mathrm{N}$ source (no AA vs no $A A+P A$ treatments) did not significantly increase microbial yield in all the species, except for $P$. bryantii $\mathrm{B}_{1} 4$ which showed $8 \%$ increase in yield from 27.4 to $29.7 \mathrm{~g}$ $\mathrm{kg}^{-1}$ (Figure 3). Microbial yields in treatments where phenyl acids were added to a medium that had complete AA mixture with aromatic AA omitted (MAR+PA treatment) were $30.9,29.6,28.0$ and 26.8 for $P$. bryantii $\mathrm{B}_{1} 4, R$. albus SY3, $R$. flavefaciens 17 and $P$. xylanivorans JK170, respectively, none of which was significantly different from MAR treatment (Figure 3).

\section{DISCUSSION}

The effects of aromatic AA and phenyl acids on some species of rumen bacteria provided with Xylan, in form of
OSX, as the only energy source were assessed. Due to the complex chemical nature of xylan, a variety of ruminal microorganisms are needed to hydrolyze and metabolize these polysaccharides (Hespell and Whitehead, 1990), although there are few exceptions. However, differences have been reported between strains and species of ruminal bacteria in their abilities to digest isolated xylans or xylans that are present in intact forages (Dehority, 2003; Hespell and Whitehead, 1990).

\section{Growth and sugar utilization}

There were varied growth responses in the present study where some rumen bacteria were able to grow, albeit to different extents, while others could not (Figures 1 and 2), perhaps due to inability to utilise xylan. Previous experiment by Hespell et al. (1987) comparing the abilities of various ruminal bacteria to degrade xylans in a Tryticase/yeast extract containing medium observed that $B$. fibrisolvens strain ARD22a could not grow at all on xylan substrates, while strains $\mathrm{H} 17 \mathrm{c}$ and $\mathrm{S} 2$ showed little growth. They also noted that although $R$. albus $7, R$. flavefaciens (C94 and FD1) and F. succinogenes S85 appeared to ferment xylan to some extent, only small amounts were utilized, and therefore little or no growth occurred. Studies by Jun et al. (2003) and Yoshida et al. (2010) clearly indicate that $F$. succinogenes S85 has xylanase genes that enable production of enzymes that can degrade xylan.

Earlier studies by Dehority (1965) showed that although major cellulolytic ruminal bacteria (that is, $F$. succinogenes, $R$. albus and $R$. flavefaciens) often can degrade hemicelluloses, only a few strains are capable of growing on these substrates. Some $R$. flavefaciens strains have been shown to utilise more than $50 \%$ of the pentose sugar present in bromegrass cell walls, but others showed little or no detectable pentose utilization (Dehority et al., 1967; Dehority, 2003). The differences in sugar 

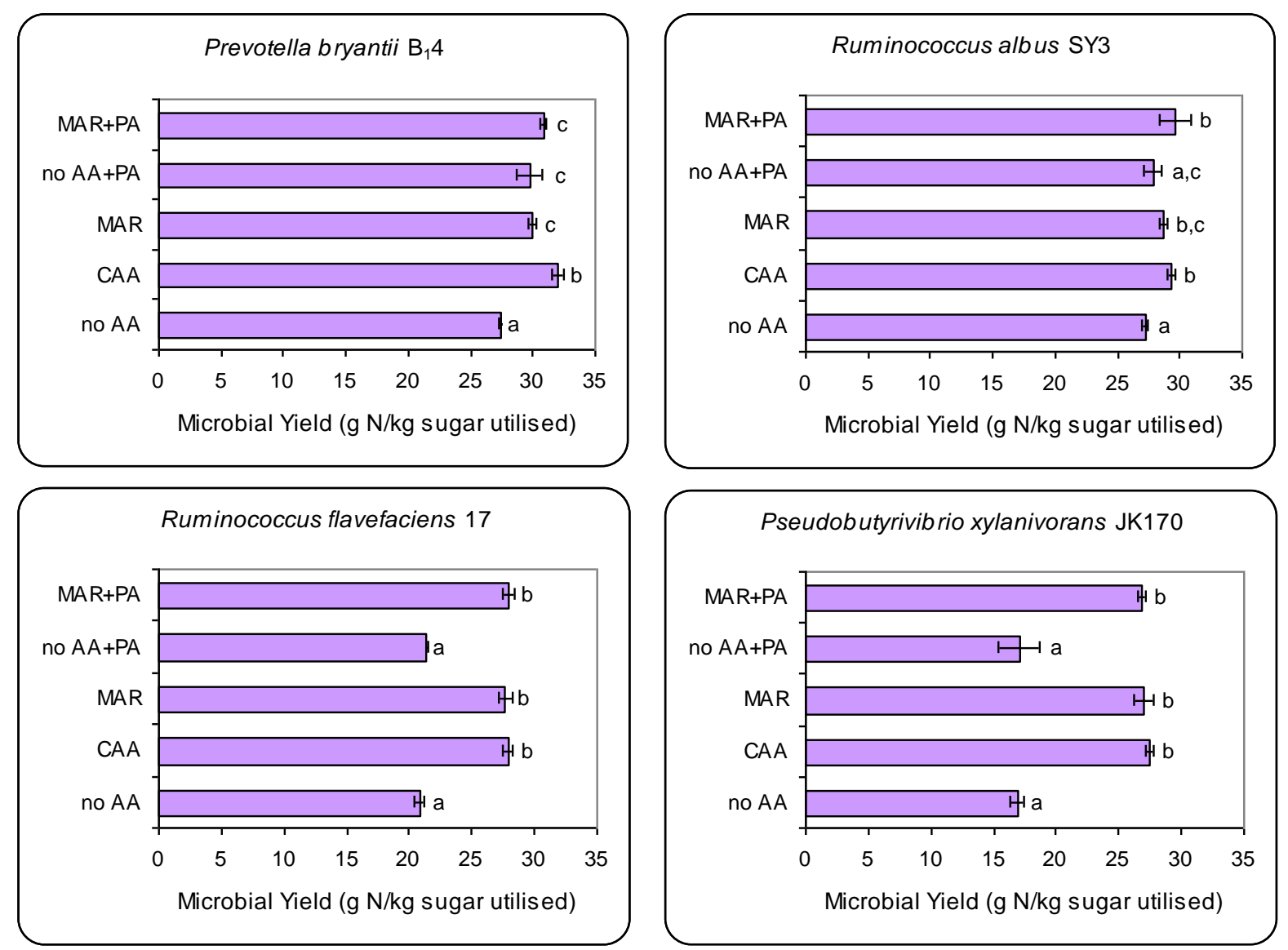

Figure 3. The influence of aromatic amino acids and phenyl acids (phenylacetic and phenylpropionic acids) on microbial yield of rumen bacteria fermenting xylan. Treatments were: no $A A=\mathrm{NH}_{3}$ only, $\mathrm{CAA}=\mathrm{complete}$ mixture of 20 amino acids, $\mathrm{MAR}=\mathrm{complete}$ amino acids mixture minus aromatic amino acids, no $\mathrm{AA}+\mathrm{PA}=\mathrm{NH}_{3}$ only $+\mathrm{PA}$, and $\mathrm{MAR}+\mathrm{PA}=$ complete amino acids mixture minus aromatic amino acids + PA. The bars represent means calculated from triplicate incubations. Bars with different letters are significantly different $(P<0.05)$.

(xylan) utilization reported in these previous studies, and also observed in the present study, can be attributed to species and strain differences.

The addition of complete AA mixtures to a medium with only ammonia as the sole $\mathrm{N}$ source seemed to stimulate the growth of bacteria able to utilise xylan (Figure 1). In terms of energetic costs, it is cheaper for the organism to utilise the readily available AA for growth. The observed growth benefit from the AA could also be related to the increased sugar utilization (Table 3 ). Previous pure culture studies have demonstrated complete AA mixtures being stimulatory to the growth of $R$. albus SY3 and $R$. flavefaciens 17 , compared to only ammonia as $\mathrm{N}$ source (Atasoglu et al., 2001). In terms of sugar utilization, $P$. bryantii $\mathrm{B}_{1} 4$ (a non-cellulolytic species), grown on a medium that contained OSX as energy source, utilised approximately $45 \%$ of OSX within $24 \mathrm{~h}$ (Miyazaki et al., 1997), which is on the lower side of the sugar utilization range observed in the present study (Table 3). Cotta (1993) observed a $46 \%$ utilization of OSX substrate by $R$. albus 7 after $24 \mathrm{~h}$ growth on a trypticase-yeast extract medium. However, Reveneau et al. (2003) noted incomplete degradation of xylan by $R$. albus and suggested that cessation of xylan degradation and growth of $R$. albus may be due to xylose accumulation. This may be the case in the present study, since sugar exhaustion cannot be the cause of cessation of growth as there was a lot of sugar left un-utilized (Table 3). Alternatively, it may be that certain linkages in OSX are either inaccessible due to the insoluble nature of the substrate or the presence of chemical linkages recalcitrant to enzymatic hydrolysis.

The deletion of aromatic AA from a complete AA mixture appeared to decrease the growth of $P$. bryantii $\mathrm{B}_{1} 4$, but had less effect on the growth of $P$. xylanivorans JK170 and the two Ruminococcus spp. (Figure 1). A similar trend is evident from the sugar utilization analysis (Table 3), suggesting that aromatic AA stimulate the utilization of xylan and growth of $P$. bryantii $\mathrm{B}_{1} 4$. Although there are no available records of aromatic AA being 
stimulatory to $P$. bryantii $\mathrm{B}_{1} 4$, there are previous reports indicating that phenylalanine benefits the growth of Ruminococcus spp. (Allison, 1965; Atasoglu et al., 2001). However, these stimulatory benefits were observed while the organisms were fermenting hexose sugar, and may explain the lack of effects on the Ruminococcus spp. in the present study where pentose sugar was the only source of energy.

The addition of phenyl acids, to no AA and MAR treatments, seem to have benefited more $P$. bryantii $B_{1} 4$, in terms of growth and xylan utilization, and to a lesser extent $R$. albus SY3 (Figure 1 and Table 3). Prevotella bryantii $\mathrm{B}_{1} 4$ have been shown to utilise more efficiently xylo-oligosaccharides and xylan fragments (Miyazaki et al., 1997). Since the addition of phenyl acids appears to have remedied the effects of aromatic AA deletion in $P$. bryantii $\mathrm{B}_{1} 4$, as shown by increased growth and sugar utilization, it may well be that the bacterium was able to utilise the phenyl acids to synthesize these AA. Several previous studies indicated that phenylalanine or its precursors, PAA and PPA, stimulated growth of Ruminococcus spp. (Allison, 1965; Stack et al. 1983; Stack and Cotta, 1986; Morrison et al., 1990). Indeed, one of the widely recognized cellulolytic organisms, $R$. albus, has a requirement for phenyl acids (PAA and PPA) for optimum growth and cellulose degradation (Hungate and Stack, 1982; Morrison et al., 1990; Reveneau et al., 2003). However, when R. albus 8 and R. flavefaciens FD1 were grown in a medium that contained Trypticase/yeast extract and wheat straw or hydrogen peroxide treated wheat straw, neither bacterial growth nor DM disappearance was affected by addition of PAA and PPA (Odenyo et al., 1991).

A study by Reveneau et al. (2003) on the possible effects of phenyl acids (PAA and PPA) or other components of rumen fluid on xylan degradation and growth of $R$. albus showed that the addition of phenyl acids (or other components present in rumen fluid) had no influence on xylan (OSX and Birchwood) degradation or the growth of any of the $R$. albus strains $(8,7$ and B199) tested. In their study they recorded $49 \%$ xylan degradation after $24 \mathrm{~h}$ incubation of $R$. albus 8 cultures supplemented with phenyl acids. It has been suggested (Allison, 1965 and 1969; Stack et al., 1983) that since phenyl acids, notably PAA, are usually present in the rumen, it may be more economical energetically for the rumen microorganisms to use these acids in the biosynthesis of aromatic AA, notably phenylalanine, than to synthesize the carbon skeleton from carbohydrate or other substances.

Although the strain JK170 has been said to belong to P. xylanivorans (Kopecny et al., 2003), it is not a well documented organism. The ability of this organism to grow on xylan and also produce butyrate as a fermentation product, as observed in this study, explains its initial classification as $B$. fibrisolvens (a butyrate producing bacterium). While it appeared to benefit from the complete AA mixture, neither the deletion of aromatic AA nor the supply of phenyl acids influenced the xylan utilization and growth of this organism (Figure 1 and Table 3).

\section{Volatile fatty acids (VFA) and ammonia}

Although the degradation of xylan by numerous ruminal bacteria have been observed, information on the type of fermentation products made is not often reported. In the present study, the main VFA produced during degradation of xylan by all the bacteria is acetate, and butyrate was only produced by $P$. xylanivorans JK170 (Table 1). Most known species of $B$. fibrisolvens produce butyrate (Stewart et al., 1997). Acetate and butyrate have been mentioned as the main fermentation acids of Butyrivibrio spp. (Miller and Wolin, 1979).

Pentose sugars can be metabolized by the transketolase and transaldolase reactions of the pentose cycle or by a phosphorolytic cleavage (phospho-ketolase) (Russell, 2002). Since acetate is the main product formed from xylan utilization by the bacterial cultures, it is likely that phospho-ketolase pathway for pentose metabolism might have predominated (Hespell et al., 1987). A study by Hespell et al. (1987) on ruminal bacteria grown in a trypticase/yeast extract medium containing OSX indicated two of the three strains of $B$. fibrisolvens (that is, AcTF2 and D1) produced acetate and butyrate, while the third strain (49) produced butyrate and no acetate. All three strains did not produce propionate, succinate or valerate, but all produced formate. From the same study, $R$. flavefaciens C94 did not also produce propionate, butyrate and valerate, but produced acetate and formate. Their study recorded VFA yields (expressed as moles of acid formed per mole of pentose fermented) that ranged between 0.48 and 1.36 .

The increase in acetate production observed in the present study across all the species provided with complete mixtures of AA (Table 1) may be due to increased utilization of xylan as shown by the data in Table 3. The growth benefits derived from the supply of AA by rumen microbes observed earlier could have resulted in increased fermentation/utilization of the energy (xylan) substrate, probably leading to the increased yields of acetate. Moreover, the breakdown of the AA may have also contributed to the increase in acetate and butyrate. The reasons behind the observed decrease in acetate yields, as a result of aromatic AA deletion from a complete mixture, in $P$. bryantii $\mathrm{B}_{1} 4$ and $R$. albus SY3 cultures is not clear. However, it is speculated that such a decrease may be due to a reduction in substrate utilization, at least for $P$. bryantii $B_{1} 4$. The supply of phenyl acids seems to induce $P$. bryantii $\mathrm{B}_{1} 4, R$. albus SY3 and $P$. xylanivorans JK170 to increase acetate production (Table 1). Whether the observed effects are due to the influence of phenyl acids 
on microbial growth and enzymic activities or a microbesubstrate interaction or both is not clear.

As expected, the treatments where complete mixtures of AA were supplied recorded highest concentrations of ammonia production (Table 2). Deamination of AA in the rumen by various ruminal bacteria leading to production of significant amounts of ammonia has long been established (El-shazly, 1952; Allison, 1969; Leng and Nolan, 1984; Wallace et al., 1997). Both Prevotella and Butyrivibrio spp. are involved in the breakdown of AA (other than those directly incorporated during cell growth) to ammonia (Wallace et al., 1997). The high ammonia production observed in $P$. bryantii $\mathrm{B}_{1} 4$ (Table 2) may be due to the ability of this organism to break down AA. Prevotella xylanivorans JK170, however, appears to be less active. Ruminococcus spp. are not generally regarded as AA fermentors and it is unclear as to why there is ammonia production.

The observed reduction in ammonia production when aromatic AA are omitted from a complete mixture (Table 2) could have been due to a reduction in microbial growth rate, at least for $P$. bryantii $\mathrm{B}_{1} 4$, leading to a slow down in AA breakdown. Phenyl acids addition caused significant reduction in ammonia production in $P$. bryantii $\mathrm{B}_{1} 4$ and $R$. albus SY3 cultures (Table 2), indicating an influence either on the organisms' AA breakdown or uptake of intact AA or maybe both. It may well be that phenyl acids stimulated the organisms to utilise more ammonia, although the mechanism for such stimulation is not clear.

\section{Microbial yield}

The microbial yield observed in treatments where AA were supplemented in $P$. bryantii $\mathrm{B}_{1} 4$ and $R$. albus SY3 were comparable to the mean yield $(30 \mathrm{~g}$ microbial $\mathrm{N} / \mathrm{Kg}$ organic matter digested) reported by ARC (1980). However, yields for $R$. flavefaciens 17 and $P$. xylanivorans JK170 were lower by between 7 and $10 \%$. Microbial yields in treatments that had only ammonia were approximately $9 \%$ lower that the ARC (1980) value for $P$. bryantii $\mathrm{B}_{1} 4$ and $R$. albus SY3; and 31 and $44 \%$ lower for $R$. flavefaciens 17 and $P$. xylanivorans JK170, respectively. The growth yield of all species was stimulated by the supply of complete mixtures of AA (Figure 3), indicating benefit derived from the AA. Previous pure culture studies also showed AA being stimulatory to the growth yield of $R$. albus SY3 and $R$. flavefaciens 17 (Atasoglu et al., 2001). Also, several experiments conducted with mixed ruminal microorganisms confirm the stimulatory effects of $A A$ on microbial growth (Argyle and Baldwin, 1989; Atasoglu et al., 1999; Kajikawa et al., 2002; Atasoglu et al., 2003).

Although the deletion of aromatic AA from a complete mixture did not have significant influence on microbial yield for all the bacteria, there was about $7 \%$ decrease in the yield of $P$. bryantii $\mathrm{B}_{1} 4$, indicative of the susceptibility of this organism to omission of these acids. Also, whereas the supply of phenyl acids did not significantly increase yields in all the species, the small benefits observed were greatest for $P$. bryantii $\mathrm{B}_{1} 4$ and $R$. albus SY3 (Figure 3), strengthening the earlier observations in growth and sugar utilization results.

The microbial yields (g TCN kg-1 sugar utilised) observed in the present study ranged between 17 and 31 (Figure 3). Hespell et al. (1987) reported cell yields (expressed as $\mathrm{g}$ of cell protein formed per mole of pentose fermented after $18 \mathrm{~h}$ of growth) of 1.1, 5.4, 3.6, 6.0, and 9.5 for $R$. flavefaciens C94 and FD1, F. succinogenes, $B$. fibrisolvens $\mathrm{H} 17 \mathrm{c}$ and $\mathrm{X} 10 \mathrm{C} 34$, respectively, using OSX as energy source.

Other strains of $B$. fibrisolvens that is, $12, A 38$ and R28 showed higher cell yields of 18.8, 22.7 and 24.0, respectively. Moreover, they also observed that although some organisms (for example, $B$. fibrisolvens HI1C and $R$. flavefaciens C94) appeared to ferment the xylans, cell yields were not as high proportionately (Hespell et al., 1987). On the basis of the final optical densities obtained, Cotta (1993) established that $P$. ruminicola converted xylooligosa-ccharides, prepared from OSX, into microbial cell mass most efficiently, while $B$. fibrisolvens was least efficient.

\section{Conclusions}

There was varied response, between species and within strains in the same species, in the growth of the ruminal microorganisms studied, perhaps influenced by ability to utilize xylan. $P$. bryantii $\mathrm{B}_{1} 4, R$. albus $\mathrm{SY} 3, R$. flavefaciens 17 and $P$. xylanivorans JK170 grew well, whereas $F$. succinogenes S85, $R$. flavefaciens FD1, and $B$. fibrisolvens JW11 could not grow. The supply of a complete mixture of complete mixtures of $A A$ to the ruminal bacteria, $P$. bryantii $\mathrm{B}_{1} 4, R$. albus SY3, $R$. flavefaciens 17, and $P$. xylanivorans JK170, stimulated xylan utilization, VFA production and microbial yield.

The deletion of aromatic AA from a complete mixture appears to affect most $P$. bryantii $\mathrm{B}_{1} 4$ in terms of growth, yield, VFA production and xylan utilization. The supply of phenyl acids (PAA and PPA) was able to reduce the effects of aromatic amino deletion in the case of $P$. bryantii $\mathrm{B}_{1} 4$. However, aromatic $A \mathrm{~A}$ and phenyl acids do not seem to greatly influence xylan utilization and growth yield of $R$. albus SY3, $R$. flavefaciens $17, P$. xylanivorans JK170, $N$. frontalis RE1 and $P$. communis $P$. The results suggest that supplementation of complete mixture of $A A$ is beneficial in stimulating bacterial growth in the rumen.

\section{ACKNOWLEDGEMENT}

We thank the Commonwealth Scholarship Commission in the United Kingdom for the support to AYG. The analytica 
analytical skills of Maureen Annand are gratefully acknowledged.

\section{REFERENCES}

Allison MJ (1965). Phenylalanine biosynthesis from from phenylacetic acid by anaerobic bacteria from the rumen. Biochem. Biophys. Res. Commun. 18:30-35.

Allison MJ (1969). Biosynthesis of amino acids by ruminal microorganisms. J. Anim. Sci. 29:797-807.

Amin MR, Onodera R (1997). Synthesis of phenylalanine and production of other related compounds from phenylpyruvic acid and phenylacetic acid by ruminal bacteria, protozoa, and their mixture in vitro. J. Gen. Appl. Microbiol. 43:9-15.

ARC (1980). The nutrient requirements of ruminant livestock. Agricultural Research Council (ARC). Commonwealth Agricultural Bureaux, UK. $351 \mathrm{p}$.

Argyle JL, Baldwin RL (1989). Effects of amino acids and peptides on rumen microbial growth yields. J. Dairy Sci. 72:2017-2027.

Atasoglu C, Guliye AY, Wallace RJ (2003). Use of a deletion approach to assess the amino acid requirements for optimum fermentation by mixed microorganisms from the sheep rumen. Anim. Sci. 76:147-153.

Atasoglu C, Newbold CJ, Wallace RJ (2001). Incorporation of $\left[{ }^{15} \mathrm{~N}\right]$ ammonia by the cellulolytic ruminal bacteria Fibrobacter succinogenes BL2, Ruminococcus albus SY3, and Ruminococcus flavefaciens 17. Appl. Environ. Microbiol. 67:819-2822.

Atasoglu C, Valdes C, Newbold CJ, Wallace RJ (1999) Influence of peptides and amino acids on fermentation rate and de novo synthesis of amino acids by mixed micro-organisms from the sheep rumen. $\mathrm{Br}$. J. Nutr. 81:307-314.

Bradford M (1976). A rapid and sensitive method for the quantitation of microgram quantities of protein utilizing the principle of protein-dye binding. Analyt. Biochem. 72:248-253.

Chen G, Strobel HJ, Russell JB, Sniffen CJ (1987). Effect of hydrophobicity on utilization of peptides by ruminal bacteria in vitro. Appl. Environ. Microbiol. 53:2021-2025.

Cotta MA (1993). Utilization of xylooligosaccharides by selected ruminal bacteria. Appl. Environ. Microbiol. 59:3557-3563.

Cotta MA, Russell JB (1982). Effect of peptides and amino acids on efficiency of rumen bacterial protein synthesis in continuous culture. $\mathrm{J}$. Dairy Sci. 65:226-234.

Cruz Soto R, Muhammed SA, Newbold CJ, Stewart CS, Wallace RJ (1993). Influence of peptides, amino acids and urea on microbial activity in the rumen of sheep receiving grass hay and on the growth of rumen bacteria in vitro. Anim. Feed Sci. Technol. 49:151-161.

Dehority BA (1965). Degradation and utilization of isolated hemicellulose by pure cultures of cellulolytic rumen bacteria. J. Bacteriol. 89:1515-1520.

Dehority BA (1967). Rate of isolated hemicellulose degradation and utilization by pure cultures of rumen bacteria. Appl. Microbiol. 15:987993.

Dehority BA (2003). Rumen Microbiology. Nottingham University Press. $372 \mathrm{p}$.

El-Shazly K (1952). Degradation of protein in the rumen of the sheep. II. The action of rumen micro-organisms on amino acids. Biochem. J. 51:647-53

Guliye AY, Atasoglu C, Wallace RJ (2005) Assessment of amino acid requirements for optimum fermentation of xylan by mixed microorganisms from sheep rumen. Anim. Sci. 80:353-360.

Guliye AY, Wallace RJ (2007). Effects of aromatic amino acids, phenylacetate and phenylpropionate on fermentation of xylan by the rumen anaerobic fungi, Neocallimastix frontalis and Piromyces communis. J. Appl. Microbiol. 103:924-929.

Hespell RB, Whitehead TR (1990). Physiology and genetics of xylan degradation by gastrointestinal tract bacteria. J. Dairy Sci. 73:30133022.

Hespell RB, Wolf R, Bothast RJ (1987). Fermentation of xylans by Butyrivibrio fibrisolvens and other ruminal bacteria. Appl. Environ. Microbiol. 53:2849-2853.
Hobson PN (1969). Rumen bacteria. Meth. Microbiol. 3B:133-149.

Hungate RE, Stack RJ (1982). Phenylpropanoic acid: growth factor for Ruminococcus albus. Appl. Environ. Microbiol. 44:79-83.

Jun HS, Ha JK, Malburg Jr LM, Verrinder Gibbins AM, Forsberg CW (2003). Characteristics of a cluster of xylanase genes in Fibrobacter succinogenes S85. Can. J. Microbiol. 49:171-180.

Kajikawa H, Mitsumori M, Ohmomo S (2002). Stimulatory and inhibitory effects of protein amino acids on growth rate and efficiency of mixed ruminal bacteria. J. Dairy Sci. 85:2015-22.

Kopecny J, Zorec M, Mrazek J, Kobayashi Y, Marinsek-Logar R (2003). Butyrivibrio hungatei sp. nov. and Pseudobutyrivibrio xylanivorans sp. nov., butyrate-producing bacteria from the rumen. Int. J. Syst. Evol. Microbiol, 53:201-209.

Kristensen S (1974). Ruminal biosynthesis of aromatic amino acids from arylacetic acids, glucose, shikimic acid and phenol. Br. J. Nutr. 31:357-65.

Leng RA, Nolan JV (1984). Nitrogen-metabolism in the rumen. J. Dairy Sci. 67:1072-1089.

Miller TL, Wolin MJ (1979). Fermentations by saccharolytic intestinal bacteria. Amer. J. Clin. Nutr. 32:164-172.

Miyazaki K, Martin JC, Marinsek-Logar R, Flint HJ (1997). Degradation and Utilization of Xylans by the Rumen AnaerobePrevotella bryanti (formerly $P$. Ruminicola subsp. brevis) $\mathrm{B}_{1} 4$. Anaerobe 3:373-381.

Morrison M, Mackie RI, Kistner A (1990). 3-Phenylpropanoic acid improves the affinity of Ruminococcus albus for cellulose in continuous culture. Appl. Environ. Microbiol. 56:3220-3222.

Odenyo AA, Mackie RI, Fahey Jr GC, White BA (1991). Degradation of wheat straw and alkaline hydrogen peroxide-treated wheat straw by Ruminococcus albus 8 and Ruminococcus flavefaciens FD-1. J. Anim. Sci. 69:819-826.

Onoda A, Kobayashi Y, Hoshino S (1996). Effects of amino acids on the growth of an anaerobic rumen fungus Neocallimastix sp $\mathrm{N} 13$ Reprod. Nutr. Dev. 36:311-320.

Orpin CG, Greenwood Y (1986). Nutritional and germination requirements of the rumen chytridiomycete Neocallimastix frontalis. Trans. Br. Mycol. Soc. 86:103-109.

Reveneau C, Adams SE, Cotta MA, Morrison M (2003). Phenylacetic and phenylpropionic acids do not affect xylan degradation by Ruminococcus albus. Appl. Environ. Microbiol. 69:6954-6958.

Russell JB (2002). Rumen microbiology and its role in ruminant nutrition. Cornell University Press, Ithaca (USA), 118p.

Scott TW, Ward PF, Dawson RM (1964). The formation and metabolism of phenyl-substituted fatty acids in the ruminant. Biochem. J. 90:1224.

Stack RJ, Cotta MA (1986). Effect of 3-phenylpropanoic acid on growth of and cellulose utilization by cellulolytic ruminal bacteria. Appl. Environ. Microbiol. 52:209-210.

Stack RJ, Hungate RE, Opsahl WP (1983). Phenylacetic acid stimulation of cellulose digestion by Ruminococcus albus 8. Appl. Environ. Microbiol. 46:539-544.

Stewart CS, Flint HJ, Bryant MP (1997). The rumen bacteria. In Hobson PN, Stewart CS (eds) The Rumen Microbial Ecosystem. Blackie Academic and Professional, London, pp. 10-72.

Wallace RJ, Onodera R, Cotta M (1997). Metabolism of nitrogen containing compounds. In Hobson PN, Stewart CS (eds) The Rumen Microbial Ecosystem. Blackie Academic and Professional, London, pp. 283-328.

Yoshida S, Mackie RI, Cann IKO (2010). Biochemical and Domain Analyses of FSUAxe6B, a Modular Acetyl Xylan Esterase, Identify a Unique Carbohydrate Binding Module in Fibrobacter succinogenes S85. J. Bacteriol. 192(2):483-493. 